FAU-TP3-98/5

hep-th/9803177

\title{
Structure and Dynamics of Monopoles in Axial-Gauge QCD*
}

\author{
O. Jahn and F. Lenz \\ Institut für Theoretische Physik III \\ Universität Erlangen-Nürnberg \\ Staudtstraße 7 \\ D-91058 Erlangen, Germany
}

(24 March 1998)

\begin{abstract}
An investigation of singular fields emerging in the process of transforming QCD to the axial gauge is presented. The structure of the singularities is analyzed. It is shown that apart from well known neutral magnetic monopole singularities, the field configurations also exhibit singularities in their charged and transverse components. This complex singularity structure guarantees finite non-Abelian field strength and thus finite action if expressed in terms of gauge fixed fields. The magnetic monopoles are characterized by two charges which are shown to determine the Pontrjagin index. Qualitative dynamical aspects of the role of the monopoles are discussed. It is argued that the entropy associated with monopoles increases with decreasing temperature and that the coupling to quantum fluctuations favors monopole-antimonopole binding.
\end{abstract}

*Copyright 1998 by The American Physical Society. 


\section{INTRODUCTION}

A fundamental and possibly far reaching difference in the formal structure of QED and QCD emerges when formulating these gauge theories in terms of physical, unconstrained variables. While this can be achieved in QED by a global - for all field configurations valid - choice of variables, this is possible in QCD only at the expense of introducing coordinate singularities and thus of including singular gauge field configurations in such gauge fixed formulations [1]. It is tempting to associate the vastly different physical properties of these two theories with this basic geometrical difference. Formation of Gribov horizons [2] or condensation of magnetic monopoles [3, 团 represent two prominent attempts in which the emergence of confinement is linked directly to the presence of singularities in gauge fields after gauge fixing. It appears that QCD lattice calculations [5,6] confirm the dynamical picture of confinement via the dual Meissner effect. Conclusive results have been obtained within the "maximal Abelian gauge". It is, however, not unambiguously established that these mechanisms are operative also in other formulations of Abelian projected QCD, and the relation to mechanisms for confinement in fundamentally different gauges, such as the Coulomb gauge, is not understood. Lattice calculations also seem to support the hypothesis of "Abelian dominance" [7] according to which long-range phenomena and specifically confinement are dominated by the "neutral" components of the gauge fields. For instance, contributions of the charged components to the string tension seem to be negligible [8]. While favored in lattice calculations, the maximal Abelian gauge is not particularly suited for explicit analytical investigations. Although selected analytical results have been obtained, in particular concerning the connection between monopoles and instantons [9, 10], a complete resolution of the maximal Abelian gauge condition is not possible nor can the singular field configurations be classified systematically. In this work we will present an analytical study of the structure and the dynamical role of singular field configurations within the modified axial (temporal) gauge representation of QCD. The reason for this particular choice is primarily technical; it is the only case where complete gauge fixing can be explicitly performed and by which a representation of QCD can be achieved in terms of unconstrained variables only. The resulting formulation has remarkable properties which are useful for clarification of both the formal structure and the dynamical implications of singular field configurations. The crucial step in the gauge fixing procedure at which singularities are generated is the diagonalization of the (untraced) Polyakov loop. The trace thereof is the order parameter characterizing the confined and deconfined phases and its values determine the presence of singularities. Furthermore, in this gauge, this order parameter is represented by an elementary rather than a composite field.

Gauge fixing by which coordinate singularities are generated is achieved through gauge transformations. Therefore, gauge invariant quantities associated with fields which become singular by gauge fixing must remain finite. This determines to a large extent the basic structure of singular field configurations. In particular singularities in the gauge field must not show up in the (non-Abelian) field strength and thus singularities necessarily occur in neutral and charged components of the gauge field simultaneously. Abelian dominance is not an immediate consequence of the singular structure of these field configurations. This elementary discussion is carried out in detail in Sec. II for gauge choices which involve diagonalization of the Polyakov loop. It is shown that in these gauges the monopoles carry, 
apart from the magnetic charge, an additional charge. This charge assumes values in the center of the gauge group and depends on the eigenvalues of the Polyakov loop. With gauge invariant quantities not affected by the presence of singularities in the gauge fields it is possible to remove those parts of space time where the gauge fields become singular and to account for the singularities by appropriate boundary conditions - a procedure similar in spirit to the Yang-Wu construction [11]. This program is explicitly carried out in Sec. [II for axial gauge QCD and, as a particular consequence, the Dirac quantization condition for charged fields moving in the background of singular fields is obtained. In axial gauge QCD, variables characterizing the orientation of the Polyakov loops have been eliminated. Therefore the singularities must be determined completely by the gauge invariant eigenvalues of the Polyakov loops. Explicit expressions in terms of these eigenvalues for the gauge fixed fields close to the singularities are given in Sec. IV. As a byproduct, this discussion will also show that the singularities have additional structures not determined by topological arguments. For instance singularities with continuously variable strength may occur in transverse Abelian magnetic fields. In general, emergence of singularities in the gauge fixing procedure does not point to any peculiar dynamics. However there are circumstances in which the presence of singularities is actually required. This issue is discussed in Sec. $\mathrm{V}$ where it will be shown that a non-vanishing topological charge associated with a gauge fixed field configuration is possible only if this configuration is singular. With the gauge fixed fields satisfying trivial boundary conditions, the topological charge receives contributions exclusively from the surface of those parts of space time which had to be removed due to the presence of singularities. In this way, a relation between topological charge and monopole charges can be established. (In a formulation involving singular non-Abelian field strengths, this relation has been given in Ref. [12].) Apart from a short summary, the concluding section contains a qualitative discussion of the physics content of the formalism developed up to this point. We do not carry out any dynamical calculation but rather interpret well known properties of QCD in terms of monopole dynamics and address specifically the issue of monopole condensation and its possible relevance for the properties of the confined and deconfined phases.

\section{SINGULARITIES FROM DIAGONALIZATION OF POLYAKOV LOOPS}

In this section we discuss the occurrence of singular gauge fields in the process of gauge fixing. We assume that the gauge fixing procedure involves diagonalization of the Polyakov loop as is the case in axial gauge. The focus of this discussion will be on the singularity structure of the fields after gauge fixing.

Throughout this work we consider SU(2) QCD at finite temperature, i.e., in Euclidean space with compact imaginary time direction of length $\beta$. The gauge potentials are assumed to be periodic up to a gauge transformation in time,

$$
A_{\mu}(\beta, \mathbf{x})=U(\mathbf{x})\left(A_{\mu}(0, \mathbf{x})+\frac{1}{i g} \partial_{\mu}\right) U^{\dagger}(\mathbf{x}),
$$

continuous before gauge fixing and to approach pure gauges at spatial infinity. The (untraced) Polyakov loops 


$$
P(\mathbf{x})=P \exp \left\{i g \int_{0}^{\beta} d x_{0} A_{0}\left(x_{0}, \mathbf{x}\right)\right\} U(\mathbf{x}) \equiv \exp \left\{i g \beta a_{0}^{c}(\mathbf{x}) \tau^{c}\right\}
$$

are of particular importance. Their definition contains the transition function $U(\mathbf{x})$ to account for the non-periodicity of the gauge fields. In this way, the eigenvalues of $P(\mathbf{x})$ are invariant also under non-periodic gauge transformations. The Polyakov loops are parametrized by the fields $a_{0}^{c}(\mathbf{x})$ whose modulus satisfies $0 \leq a_{0}(\mathbf{x}) \leq \pi / g \beta$. This restriction does not spoil the continuity of $a_{0}$ as follows from the continuity of $\operatorname{tr} P=2 \cos g \beta a_{0}$. The restriction, however, introduces discontinuities in derivatives similar to those appearing in the transition from Cartesian to polar coordinates. For the following topological considerations, continuity is sufficient. The discontinuities will be taken into account in the discussion of the detailed structure of the singularities in Sec. [II]. In the absence of quarks, the expectation value of the (traced) Polyakov loops serves as order parameter for characterization of the confined and deconfined phases. In the axial (temporal) gauge representation, the Polyakov loop variables $a_{0}^{c}$ appear as elementary rather than composite degrees of freedom. This representation of QCD is obtained as a result of the following gauge transformation performed in three steps [13],

$$
\Omega(x)=\Omega_{\mathrm{D}}(\mathbf{x}) \exp \left\{-i g x_{0} a_{0}^{c}(\mathbf{x}) \tau^{c}\right\} P \exp \left\{i g \int_{0}^{x_{0}} d t A_{0}(t, \mathbf{x})\right\} .
$$

In the presence of the third factor only, the gauge transformation would eliminate $A_{0}$ completely. The second term reintroduces zero mode fields, and is constructed such that the transformed gauge fields are strictly periodic in time. Finally $\Omega_{\mathrm{D}}$ diagonalizes these zero mode fields or, equivalently, the Polyakov loops of Eq. (2),

$$
\Omega_{\mathrm{D}}(\mathbf{x}) P(\mathbf{x}) \Omega_{\mathrm{D}}^{\dagger}(\mathbf{x})=e^{i g \beta a_{0}(\mathbf{x}) \tau_{3}} .
$$

As a result of the gauge fixing transformation (3), the time component of the gauge field is eliminated apart from a diagonal zero mode field,

$$
\Omega(x)\left(A_{0}(x)+\frac{1}{i g} \partial_{0}\right) \Omega^{\dagger}(x)=a_{0}(\mathbf{x}) \tau_{3} .
$$

The diagonalization (4) can also be interpreted as a choice of coordinates in color space. With Eq. (4), the color three direction is chosen to be that of the Polyakov loop at given $\mathbf{x}$. This diagonalization is the crucial element of the gauge fixing procedure by which singular field configurations emerge. It is most easily studied explicitly by introducing polar $(\theta)$ and azimuthal $(\varphi)$ angles in color space,

$$
\begin{aligned}
\tilde{a}^{c}(\mathbf{x}) \tau^{c} & =\tilde{\mathbf{a}} \cdot \boldsymbol{\tau}=\sin \left(g \beta a_{0}^{c}(\mathbf{x}) \tau^{c}\right) \\
& =\sin \left(g \beta a_{0}(\mathbf{x})\right)\left(\begin{array}{cc}
\cos (\theta(\mathbf{x})) & e^{-i \varphi(\mathbf{x})} \sin (\theta(\mathbf{x})) \\
e^{i \varphi(\mathbf{x})} \sin (\theta(\mathbf{x})) & -\cos (\theta(\mathbf{x}))
\end{array}\right)
\end{aligned}
$$

Note that $\tilde{\mathbf{a}}$ is a continuous function of the (untraced) Polyakov loop $P$. With this choice of coordinates, the matrix $\Omega_{\mathrm{D}}(\mathbf{x})$ can be represented as 


$$
\Omega_{\mathrm{D}}(\mathbf{x})=\left(\begin{array}{cc}
e^{i \varphi(\mathbf{x})} \cos (\theta(\mathbf{x}) / 2) & \sin (\theta(\mathbf{x}) / 2) \\
-\sin (\theta(\mathbf{x}) / 2) & e^{-i \varphi(\mathbf{x})} \cos (\theta(\mathbf{x}) / 2)
\end{array}\right) .
$$

Consequences related to the ambiguities in the definition of $\Omega_{\mathrm{D}}$ will be discussed later. To improve readability, we suppress the arguments of $\theta$ and $\varphi$ in the following.

In general, diagonalization, or equivalently the choice of coordinates, is not everywhere well defined and consequently (coordinate) singularities occur in the associated transformations of the gauge fields. Starting from an everywhere-regular gauge field $A_{i}$, the transformed field

$$
A_{i}^{\prime}(x)=\Omega_{\mathrm{D}}(\mathbf{x}) A_{i}(x) \Omega_{\mathrm{D}}^{\dagger}(\mathbf{x})+s_{i}(\mathbf{x})
$$

with

$$
s_{i}(\mathbf{x})=\Omega_{\mathrm{D}}(\mathbf{x}) \frac{1}{i g} \partial_{i} \Omega_{\mathrm{D}}^{\dagger}(\mathbf{x})
$$

is in general singular with $\Omega_{\mathrm{D}}$. While the homogeneous term can at most be discontinuous, the inhomogeneous term diverges in general. We decompose this inhomogeneous term into neutral $\left(\tau_{3}\right)$ and charged $\left(\tau_{ \pm}=\left(\tau_{1} \pm i \tau_{2}\right) / 2\right)$ components with respect to the color orientation of the Polyakov loop,

$$
s_{i}(\mathbf{x})=a_{i}^{\mathrm{s}}(\mathbf{x}) \tau_{3}+\left(\phi_{i}^{\mathrm{s}}(\mathbf{x}) \tau_{+}+\text {h.c. }\right),
$$

where

$$
\begin{aligned}
& a_{i}^{\mathrm{s}}(\mathbf{x})=-\frac{1}{2 g}(1+\cos \theta) \partial_{i} \varphi=-\frac{1+\cos \theta}{2 g \sin \theta} \frac{1}{\sin g \beta a_{0}} \hat{\boldsymbol{\varphi}} \cdot \partial_{i} \tilde{\mathbf{a}}, \\
& \phi_{i}^{\mathrm{s}}(\mathbf{x})=\frac{1}{2 g} e^{i \varphi}\left(\sin \theta \partial_{i} \varphi+i \partial_{i} \theta\right)=\frac{1}{2 g \sin g \beta a_{0}} e^{i \varphi}(\hat{\boldsymbol{\varphi}}+i \hat{\boldsymbol{\theta}}) \cdot \partial_{i} \tilde{\mathbf{a}}
\end{aligned}
$$

with the standard choice of unit vectors

$$
\hat{\boldsymbol{\varphi}}=\left(\begin{array}{c}
-\sin \varphi \\
\cos \varphi \\
0
\end{array}\right) \quad \text { and } \quad \hat{\boldsymbol{\theta}}=\left(\begin{array}{c}
\cos \theta \cos \varphi \\
\cos \theta \sin \varphi \\
-\sin \theta
\end{array}\right)
$$

In order to make the singularity structure manifest we have expressed $s_{i}$ in terms of the continuous field ã present before gauge fixing (cf. Eq. (6)). Singularities (poles) occur at points $\mathrm{x}^{\mathrm{N}, \mathrm{S}}$, where the Polyakov loop passes through the center of the group and does not define a direction in color space,

$$
z \equiv P\left(\mathbf{x}^{\mathrm{N}, \mathrm{S}}\right)= \pm \mathbb{1}
$$

This requirement determines a point on the group manifold $\mathrm{S}^{3}$ and thus, for generic cases, fixes (locally) uniquely the position $\mathrm{x}^{\mathrm{N}, \mathrm{S}}$. In four space the transformed gauge fields are thus singular on straight lines parallel to the time axis, i.e., they represent static singular field configurations. The static nature of the singularities is a trivial consequence of the 
static Polyakov loop which has been selected for introducing coordinates in color space. The singularities can be distinguished by the value of the Polyakov loop and we shall refer to them as north $\left(g \beta a_{0}\left(\mathbf{x}^{\mathrm{N}}\right)=0\right)$ and south $\left(g \beta a_{0}\left(\mathbf{x}^{\mathrm{S}}\right)=\pi\right)$ pole singularities according to the respective positions of the Polyakov loop on the group manifold $\mathrm{SU}(2)\left(\cong \mathrm{S}^{3}\right)$. Thus we can assign a "north-south" quantum number or charge $z$ to this singularity (i.e., the range of $z$ is the center of the group). This north-south charge should not be confused with the magnetic charge to be introduced below. In addition to poles, the field $a_{i}^{\mathrm{s}}$ also exhibits (static) string like singularities along the line $\theta=0$ representing a surface in four space. The charged gluon fields too have poles at $\mathbf{x}^{\mathrm{N}, \mathrm{S}}$ and discontinuities along the strings $\theta=0$. Although the points $\mathrm{x}^{\mathrm{N}, \mathrm{S}}$ are characterized by Eq. (13) in a gauge invariant way (by the degeneracy of two gauge invariant eigenvalues), in general those points have no particular significance in other gauges.

Before continuing with our general discussion we consider special field configurations in which color and spatial orientations are identified,

$$
\varphi(\mathbf{x})= \pm \varphi_{0} \equiv \pm \arctan \frac{y}{x}, \quad \theta(\mathbf{x})=\theta_{0} \equiv \arccos \frac{z}{r} .
$$

Thereby the singular field $a_{i}^{\mathrm{s}}(\mathbf{x})$ in Eq. (11) becomes exactly the vector potential of a Dirac monopole [14 of charge $\pm 2 \pi / g$,

$$
\mathbf{a}^{ \pm}(\mathbf{x})=\mp \frac{1}{2 g} \frac{1+\cos \theta_{0}}{r \sin \theta_{0}} \hat{\boldsymbol{\varphi}}_{0}
$$

with Abelian (neutral) magnetic field

$$
\mathbf{b}^{ \pm}=\operatorname{curl} \mathbf{a}^{ \pm}= \pm \frac{1}{2 g} \frac{\mathbf{x}}{x^{3}}
$$

Associated with the singular neutral component is a singular charged component which is given by

$$
\boldsymbol{\phi}^{ \pm}(\mathbf{x})=\frac{1}{2 g r}\left( \pm \hat{\boldsymbol{\varphi}}_{0}+i \hat{\boldsymbol{\theta}}_{0}\right) e^{ \pm i \varphi_{0}}
$$

In returning to the formal development, we introduce the Abelian field strength

$$
f_{i j}=\partial_{i} a_{j}^{\mathrm{s}}-\partial_{j} a_{i}^{\mathrm{s}}
$$

an important quantity in Abelian projected theories. It is singular at the position of the monopoles. On the other hand, the complete non-Abelian field strength built from the inhomogeneous term $s_{i}$ of Eq. (9) actually vanishes,

$$
F_{i j}[s]=\partial_{i} s_{j}-\partial_{j} s_{i}+i g\left[s_{i}, s_{j}\right]=0
$$

i.e., the singular Abelian field strength is exactly canceled by the non-Abelian contribution to $F$ generated by the singularities in the charged gluon fields.

A non-vanishing field strength can occur only at the singularities of the pure gauge field $s_{i}$. Application of the Gauss theorem shows that the non-Abelian field strength does 
not contain monopole singularities and by application of the standard (Abelian) Yang-Wu construction [11] strings can be completely avoided. The cancellation of the singularities in the Abelian field strength by the non-Abelian commutator must happen quite generally. The singular fields arise as coordinate singularities since we insist on fixing the gauge globally. In adopting a generalized Yang-Wu construction with some other (non-Abelian) gauge choice on charts which cover the neighborhood of the points $\mathrm{x}^{\mathrm{N}, \mathrm{S}}$, occurrence of any singularity can be avoided. It is important for the following that despite the singularities generated in the gauge fields the gauge fixing procedure does not affect gauge invariant quantities even at the positions of the monopoles and along the strings. This will allow us to remove these points and strings from space time at the expense of formulating appropriate boundary conditions for the gauge fields. (In a similar context, this procedure to exclude the singular points from space time has been discussed in Ref. [15].) A further consequence of the non-Abelian field strength being finite is the rigid connection between neutral and charged singular components of the gauge fields. Abelian dominance is thus not realized at the level of the singular field configurations. Instead of applying a Yang-Wu construction, one may proceed by regularizing the singular field configurations (cf. Ref. [16]). In this way the "coordinate" transformation corresponding to Eq. (3) does not necessarily remain a gauge transformation and therefore values of gauge invariant quantities like $\operatorname{tr}\left(F^{2}\right)$ do not remain invariant and may even become infinite when removing the regulator. In Ref. [16] it is argued that monopole contributions to the Abelian and non-Abelian terms in Eq. (19) cancel. In contrast to our approach, infinite non-Abelian fields appear along the strings.

Unlike the points $\mathrm{x}^{\mathrm{N}, \mathrm{S}}$ which are determined in a gauge invariant way (cf. Eq. (13)) the location of the strings is to a large extent still arbitrary since the gauge transformation $\Omega$ of Eq. (3) does not fix the gauge completely. An Abelian rotation with an $\mathbf{x}$-dependent gauge function does not affect the gauge condition (5). Complete gauge fixing can be achieved for instance by imposing a standard gauge condition on the zero modes of the neutral gluons $\left(\int_{0}^{\beta} d x_{0} A_{i}^{3}\right)$ as in Ref. [17]. For the investigation of the singular fields it is advantageous not to perform this final step in gauge fixing and to use the residual gauge freedom to change the location of the strings. Indeed, a time-independent Abelian gauge transformation corresponds to a redefinition of $\Omega_{\mathrm{D}}$,

$$
\Omega_{\mathrm{D}}(\mathbf{x}) \rightarrow e^{i \tau_{3} \psi(\mathbf{x})} \Omega_{\mathrm{D}}(\mathbf{x})
$$

with arbitrary $\psi$. The related changes in the singular gauge fields are

$$
\begin{aligned}
& a_{i}^{\mathrm{s}}(\mathbf{x}) \rightarrow a_{i}^{\mathrm{s}}(\mathbf{x})-\frac{1}{g} \partial_{i} \psi(\mathbf{x}), \\
& \phi_{i}^{\mathrm{s}}(\mathbf{x}) \rightarrow \phi_{i}^{\mathrm{s}}(\mathbf{x}) e^{2 i \psi(\mathbf{x})} .
\end{aligned}
$$

Using this ambiguity in the diagonalization of the Polyakov loop, i.e., the residual gauge freedom, strings can be deformed in space. For instance, the gauge function

$$
\psi(\mathbf{x})=-\varphi(\mathbf{x})
$$

changes a string along $\theta(\mathbf{x})=0$ into a string along $\theta(\mathbf{x})=\pi$. More generally, the gauge function which transforms a gauge field with string $\mathbf{s}_{1}(\mathbf{x})$ into a field with string $\mathbf{s}_{2}(\mathbf{x})$ can be constructed by using identities well known from magnetostatics and is given by [18] 


$$
\psi(\mathbf{x})=\int_{\Sigma\left(\mathbf{s}_{1}, \mathbf{s}_{2}\right)} \frac{\mathbf{x}-\mathbf{x}^{\prime}}{\left|\mathbf{x}-\mathbf{x}^{\prime}\right|^{3}} \cdot d^{2} \boldsymbol{\sigma}\left(\mathbf{x}^{\prime}\right),
$$

where the integral is performed over an arbitrary surface bounded by the two strings (and possibly extending to infinity).

Topological considerations [5] can be used to characterize some (but not all; cf. Sec. IV) of the properties of the singularities from a more general point of view. The residual Abelian symmetry provides the basis for this discussion. The ambiguity in the definition of $\Omega_{\mathrm{D}}$ expressed by Eq. (20) suggests one can interpret $\Omega_{\mathrm{D}}$ as an element of $\mathrm{SU}(2) / \mathrm{U}(1)$. Poles appear where the eigenvalues of $P$ are degenerate and $\Omega_{\mathrm{D}}$ is ill-defined. Due to the nontriviality of the second homotopy group $\pi_{2}(\mathrm{SU}(2) / \mathrm{U}(1))=Z$ a winding number can be assigned to this singularity. A configuration of $\Omega_{\mathrm{D}}$ on a sphere around $\mathrm{x}^{\mathrm{N}, \mathrm{S}}$ with a nonvanishing winding number is not smoothly deformable to a constant and a discontinuity will remain upon shrinking the sphere to a point. The winding number of $\Omega_{\mathrm{D}}$ around $\mathbf{x}^{\mathrm{N}, \mathrm{S}}$ can be expressed as

$$
\begin{aligned}
n & \equiv \frac{i}{4 \pi} \epsilon_{i k l} \int_{\left|\mathbf{x}-\mathbf{x}^{\mathrm{N}, \mathrm{S}}\right|=\varepsilon} d^{2} \sigma_{i} \operatorname{tr} \tau_{3} \Omega_{\mathrm{D}} \partial_{k} \Omega_{\mathrm{D}}^{\dagger} \Omega_{\mathrm{D}} \partial_{l} \Omega_{\mathrm{D}}^{\dagger} \\
& =-\frac{i}{4 \pi} \epsilon_{i k l} \int_{\left|\mathbf{x}-\mathbf{x}^{\mathrm{N}, \mathrm{S}}\right|=\varepsilon} d^{2} \sigma_{i} \partial_{k}\left(\Omega_{\mathrm{D}} \partial_{l} \Omega_{\mathrm{D}}^{\dagger}\right) .
\end{aligned}
$$

As it should be, $n$ is invariant under multiplication of $\Omega_{\mathrm{D}}$ with an (x dependent) diagonal matrix from the left. With the winding number density being a total derivative, $n$ can be different from zero only if $\Omega_{\mathrm{D}}$ is discontinuous at some point $\mathbf{x}^{0}$ on any sphere surrounding $\mathbf{x}^{\mathrm{N}, \mathrm{S}}$. These points will form a string of discontinuity emanating from $\mathbf{x}^{\mathrm{N}, \mathrm{S}}$. The discontinuity is due to the undetermined $\mathrm{U}(1)$ factor of $\Omega_{\mathrm{D}}$. When lifting a function from $\mathrm{S}^{2}$ to $\mathrm{SU}(2) / \mathrm{U}(1)$ having a non-vanishing winding-number to $\mathrm{SU}(2)$, points with an Abelian discontinuity can not be avoided [19]:

$$
\Omega_{\mathrm{D}}(\mathbf{x}) \rightarrow e^{i \psi(\mathbf{x}) \tau_{3}} \Omega_{\mathrm{D}}^{0} \quad \text { for } \quad \mathbf{x} \rightarrow \mathbf{x}^{0}
$$

Here, $\psi$ changes by an integer multiple of $2 \pi$ when the string is encircled once. We may think of this integer as a winding number assigned to the string,

$$
n^{\text {string }} \equiv \frac{\delta \psi}{2 \pi}=\frac{1}{2 \pi} \int_{C} d s_{j} \partial_{j} \psi=\frac{-i}{4 \pi} \int_{C} d s_{j} \operatorname{tr} \tau_{3} \Omega_{\mathrm{D}} \partial_{j} \Omega_{\mathrm{D}}^{\dagger},
$$

where the integrals are performed over a curve $C$ around the string. By Eq. (24), this winding number, or if there are several discontinuities the sum of the respective winding numbers, equals the winding number of the monopole $n$.

For our particular parametrization, Eq. (7), $\Omega_{\mathrm{D}}$ becomes discontinuous when approaching the curve $\theta=0$,

$$
\Omega_{\mathrm{D}}(\mathbf{x}) \rightarrow e^{i \varphi(\mathbf{x}) \tau_{3}}\left(\begin{array}{ll}
1 & 0 \\
0 & 1
\end{array}\right) \quad \text { for } \quad \theta \rightarrow 0
$$

Note that the Abelian magnetic field associated with the singular field $a_{i}^{\mathrm{s}}$ (cf. Eqs. (9, 10)), 


$$
b_{k}^{\mathrm{s}}=\epsilon_{k i j} \partial_{i} a_{j}^{\mathrm{s}}=\epsilon_{k i j} \partial_{i}\left(\frac{1}{2 i g} \operatorname{tr} \tau_{3} \Omega_{\mathrm{D}} \partial_{j} \Omega_{\mathrm{D}}^{\dagger}\right),
$$

is proportional to the integrand in Eq. (24). Thus, all topologically non-trivial point singularities give rise to magnetic monopoles with quantized charges $2 \pi n / g$. These singularities are therefore characterized by two charges, the magnetic charge and the north-south charge (cf. Eq. (13)). The emergence of two quantum numbers is a specific property of our gauge choice which involves diagonalization of an element of the group rather than the Lie algebra. The presence of two quantum numbers will be seen to be crucial for establishing a relation to the topological charge in Sec. $\mathrm{V}$.

\section{CONTINUOUS AND SINGULAR FIELDS IN AXIAL GAUGE}

In this section we construct the singular gauge field configurations in the axial gauge. To this end, we have to account for the other two factors appearing in the gauge fixing transformation of Eq. (3) besides the diagonalization $\left(\Omega_{\mathrm{D}}\right)$ of the Polyakov loops. This construction allows one to decompose the gauge field $A_{i}^{\prime}$ into regular and singular terms which separately are periodic in time. Such a decomposition is a prerequisite for formulating the (gauge fixed) path integral in the presence of singular field configurations. It is obvious that for such a sum of regular and singular terms to correspond to a continuous field before gauge fixing, the regular term has to satisfy certain conditions in the neighborhood of the singularities which will be derived now. The starting point is the continuity of the original field configuration. The first step in the gauge fixing procedure does not produce any singularities,

$$
A_{i}^{(1)} \equiv P e^{i g \int_{0}^{x_{0}} d t A_{0}(t, \mathbf{x})}\left(A_{i}+\frac{1}{i g} \partial_{i}\right)\left(P e^{i g \int_{0}^{x_{0}} d t A_{0}(t, \mathbf{x})}\right)^{\dagger}=\text { continuous, }
$$

because the path ordered integral is continuous in $\mathbf{x}$ (but not periodic in $x_{0}$ ). The remaining steps can be written as

$$
A_{i}^{\prime}=e^{-i g a_{0} x_{0} \tau_{3}} \Omega_{\mathrm{D}} A_{i}^{(1)} \Omega_{\mathrm{D}}^{\dagger} e^{i g a_{0} x_{0} \tau_{3}}+e^{-i g a_{0} x_{0} \tau_{3}} s_{i} e^{i g a_{0} x_{0} \tau_{3}}+\partial_{i} a_{0} x_{0} \tau_{3},
$$

where we have pulled the second factor in Eq. (3) through $\Omega_{\mathrm{D}}$ and inserted the singular field $s_{i}$ arising from the diagonalization (cf. Eq. (9)). We observe that the off-diagonal part of the singular field acquires a time dependent (and non-periodic) phase $2 g a_{0} x_{0}$. At the north pole, this phase vanishes and does not influence the divergent part of $s_{i}$. At the south pole, however, $g a_{0} \rightarrow \pi / \beta$, and a time-dependent (but now periodic) phase persists. Since we do not want to introduce a non-periodic singular field, we decompose the gauge field as

$$
A_{i}^{\prime}=\bar{\omega} s_{i} \bar{\omega}^{\dagger}+\hat{A}_{i}
$$

where $\bar{\omega}$ is the diagonal matrix

$$
\bar{\omega}(x)=\left(\begin{array}{cc}
\omega^{1 / 2}(x) & 0 \\
0 & \omega^{-1 / 2}(x)
\end{array}\right)
$$


with $\omega(x)$ satisfying

$$
\begin{array}{ll}
\omega(x) \rightarrow 1 & \text { at northern monopole, } \\
\omega(x) \rightarrow e^{-2 i \pi x_{0} / \beta} & \text { at southern monopole. }
\end{array}
$$

With this choice, $\hat{A}_{i}$ is finite. To investigate its discontinuity, we consider

$$
\begin{aligned}
& \Omega_{\mathrm{D}}^{\dagger} e^{i g a_{0} x_{0} \tau_{3}} \hat{A}_{i} e^{-i g a_{0} x_{0} \tau_{3}} \Omega_{\mathrm{D}} \\
& \quad=A_{i}^{(1)}+\Omega_{\mathrm{D}}^{\dagger}\left(s_{i}-e^{i g a_{0} x_{0} \tau_{3}} \bar{\omega} s_{i} \bar{\omega}^{\dagger} e^{-i g a_{0} x_{0} \tau_{3}}+\partial_{i} a_{0} x_{0} \tau_{3}\right) \Omega_{\mathrm{D}} \\
& \quad=A_{i}^{(1)}+\Omega_{\mathrm{D}}^{\dagger}\left(\begin{array}{cc}
\partial_{i} a_{0} x_{0} & \phi_{i}^{\mathrm{s}}\left(1-e^{2 i g a_{0} x_{0}} \omega\right) \\
\phi_{i}^{\mathrm{s}}\left(1-e^{-2 i g a_{0} x_{0}} \omega^{\dagger}\right) & -\partial_{i} a_{0} x_{0}
\end{array}\right) \Omega_{\mathrm{D}} .
\end{aligned}
$$

The right-hand side of this equation is continuous at the strings because there the discontinuities of $\phi_{i}^{\mathrm{s}}$ and $\partial_{i} a_{0}$ are canceled by the discontinuity of $\Omega_{\mathrm{D}}$. An expansion in terms of $g \beta a_{0}$ or $\pi-g \beta a_{0}$ yields that it is also continuous at the monopoles. On the left-hand side, we can replace $\exp \left(i g a_{0} x_{0} \tau_{3}\right)$ by $\bar{\omega}$. The final continuity requirement for $\hat{A}_{i}$ reads

$$
\Omega_{\mathrm{D}}^{\dagger} \bar{\omega}^{\dagger} \hat{A}_{i} \bar{\omega} \Omega_{\mathrm{D}}=\text { continuous. }
$$

The discontinuous factor $\Omega_{\mathrm{D}}$ implies a discontinuity in $\hat{A}_{i}$ which cannot be absorbed into a redefinition of the inhomogeneous singular terms $s_{i}$ in Eq. (31). For the following it is convenient to introduce a spherical color basis,

$$
\begin{aligned}
& \hat{A}_{i}^{1}=\frac{1}{\sqrt{2}}\left(\hat{\Phi}_{i}+\hat{\Phi}_{i}^{\dagger}\right), \\
& \hat{A}_{i}^{2}=\frac{i}{\sqrt{2}}\left(\hat{\Phi}_{i}-\hat{\Phi}_{i}^{\dagger}\right) .
\end{aligned}
$$

In this basis the continuity requirement reads

$$
\begin{aligned}
& \cos \theta \hat{A}_{i}^{3}-\sin \theta\left[e^{-i \varphi} \frac{1}{\sqrt{2}} \hat{\Phi}_{i} \omega^{\dagger}+\text { c.c. }\right]=\text { continuous } \\
& \sin \theta e^{-i \varphi} \hat{A}_{i}^{3}+(1+\cos \theta) e^{-2 i \varphi} \frac{1}{\sqrt{2}} \hat{\Phi}_{i} \omega^{\dagger}-(1-\cos \theta) \frac{1}{\sqrt{2}} \hat{\Phi}_{i}^{\dagger} \omega=\text { continuous }
\end{aligned}
$$

which determines the behavior of the fields $\hat{A}_{i}$ in the neighborhood of monopoles and strings. In particular, along the strings, Eq. (37) reduces to

$$
e^{-2 i \varphi} \hat{\Phi}_{i}=\text { continuous at the string, }
$$

a condition familiar from the treatment of the motion of a charged particle in the field of a Dirac monopole. Continuity of the original field configuration guarantees "invisibility" of strings and monopoles. These continuity requirements clearly differentiate between charged and neutral components. In particular, the neutral fields $\hat{A}_{i}^{3}$ are not affected by the presence of the strings. Abelian dominance, though not valid for the singular configurations, possibly has its origin in this distinction and thus would be a property of the fluctuating fields. On the basis of the decomposition (31), the field strength associated with singular field 
configurations is straightforwardly calculated. The definition of charged gluon fields in the spherical color basis of Eq. (36) suggests introduction, alongside the neutral component

$$
F_{i j}^{3}=\partial_{i} A_{j}^{3}-\partial_{j} A_{i}^{3}-i g\left(\Phi_{i}^{\dagger} \Phi_{j}-\Phi_{j}^{\dagger} \Phi_{i}\right)
$$

of the following charged component of the field strength,

$$
\chi_{i j}=\left(\partial_{i}+i g A_{i}^{3}\right) \Phi_{j}-\left(\partial_{j}+i g A_{j}^{3}\right) \Phi_{i} .
$$

The spatial part of the Yang-Mills Lagrangian is then given by

$$
\frac{1}{2} \sum_{a=1}^{3} F_{i j}^{a} F_{i j}^{a}=\frac{1}{2} F_{i j}^{3} F_{i j}^{3}+\chi_{i j} \chi_{i j}^{\dagger} .
$$

It is furthermore convenient to include the Polyakov loop variables $a_{0}(\mathbf{x})$ into the definition of the singular field configuration. In this way, the dependence on the original variable $A_{0}$ is completely absorbed into the singular part $\alpha_{\mu}$ defined by

$$
\begin{aligned}
\alpha_{\mu}^{3}(\mathbf{x}) & =2\left(a_{\mu}^{\mathrm{s}}(\mathbf{x})\left(1-\delta_{\mu, 0}\right)+a_{0}(\mathbf{x}) \delta_{\mu, 0}\right), \\
\alpha_{\mu}^{1}(x)+i \alpha_{\mu}^{2}(x) & =2 \omega(x) \phi_{\mu}^{\mathrm{s}}(\mathbf{x})\left(1-\delta_{\mu, 0}\right) .
\end{aligned}
$$

The total field is given by

$$
A_{\mu}^{\prime}=\alpha_{\mu}+\hat{A}_{\mu}
$$

where in axial gauge $\hat{A}_{0}=0$. The field strength is accordingly decomposed,

$$
F_{\mu \nu}[\hat{A}+\alpha]=F_{\mu \nu}[\alpha]+\hat{D}_{\mu} \hat{A}_{\nu}-\hat{D}_{\nu} \hat{A}_{\mu}+i g\left[\hat{A}_{\mu}, \hat{A}_{\nu}\right]
$$

with the covariant derivative

$$
\hat{D}_{\mu}=\partial_{\mu}+i g\left[\alpha_{\mu}\right.
$$

being defined in terms of the singular fields. For the evaluation of the field strength we observe that for $\omega(x) \equiv 1$ (cf. Eq. (33)) the singular gauge field $\alpha_{i}$ is a spatial pure gauge, i.e., the spatial field strength components vanish in this limit. This simplifies the expressions and we easily find

$$
\begin{aligned}
F_{i 0}^{3}[\alpha] & =2 \partial_{i} a_{0}(\mathbf{x}), \quad \chi_{i 0}[s]=\sqrt{2} \phi_{i}^{\mathrm{s}}(\mathbf{x})\left(-\partial_{0}+2 i g a_{0}(\mathbf{x})\right) \omega(x), \\
F_{i j}^{3}[\alpha] & =2 i g\left(\omega(x) \omega(x)^{\dagger}-1\right)\left(\phi_{i}^{\mathrm{s} \dagger}(\mathbf{x}) \phi_{j}^{\mathrm{s}}(\mathbf{x})-\phi_{j}^{\mathrm{s}}(\mathbf{x}) \phi_{i}^{\mathrm{s}}(\mathbf{x})\right), \\
\chi_{i j}[\alpha] & =\sqrt{2}\left(\phi_{j}^{\mathrm{s}}(\mathbf{x}) \partial_{i} \omega(x)-\phi_{i}^{\mathrm{s}}(\mathbf{x}) \partial_{j} \omega(x)\right) .
\end{aligned}
$$

As could be expected, these explicit formulae for the Yang-Mills field strength components display that in general no particularly large or even divergent action is associated with singular gauge field configurations, i.e., monopoles generated by gauge fixing have finite action. More subtle in these expressions for the field strength is the distinction between configurations which contain one type of singularity (north or south poles only) from configurations 
in which both types occur as a function of $\mathbf{x}$. In the former case we can choose $\omega(x)=1$ for configurations with north and $\omega(x)=\exp \left(2 i \pi x_{0} / \beta\right)$ for configurations with south pole singularities only and the action generated by such configurations can be arbitrarily small. In this case the spatial components of the associated field strength vanish, $F_{i j}=0$. If both types of singularity are present, there are necessarily spatial components which contribute to the action. As we shall see below, instantons belong to field configurations of this second type. Fluctuations around the singular fields are described by the fields $\hat{A}_{\mu}$ in Eq. (43). The covariant derivative $\hat{D}_{\mu}$ of Eq. (45) couples these two fields. The boundary conditions (37) imposed on the fluctuating fields guarantee finiteness of the total action. The relevance of these expressions for formulation of the quantum theory in the presence of singular field configurations is obvious.

Finally, we point out the residual local gauge invariance of the action

$$
S[\hat{A}+\alpha]=\frac{1}{2} \int d^{4} x \operatorname{tr}\left(F_{\mu \nu}[\hat{A}+\alpha] F_{\mu \nu}[\hat{A}+\alpha]\right) .
$$

The effect on $S$ of rotating the color components,

$$
\hat{A}_{\mu}^{\prime}(x)=e^{i \psi(\mathbf{x}) \tau_{3}} \hat{A}_{\mu}(x) e^{-i \psi(\mathbf{x}) \tau_{3}},
$$

can be compensated for by an appropriate change in the singular background field (cf. Eq. (21)),

$$
\alpha_{\mu}^{\prime}=e^{i \psi(\mathbf{x}) \tau_{3}}\left(\alpha_{\mu}+\frac{1}{i g} \partial_{\mu}\right) e^{-i \psi(\mathbf{x}) \tau_{3}} .
$$

\section{MONOPOLE PARAMETERS}

In the completely gauge fixed formulation there can be no dependence on the orientation of the Polyakov loops (or, equivalently, of ã). This is not manifest in our formulation since the expressions for the singular field configurations (11) depend explicitly on the orientation of ã. Quantities like the Abelian magnetic field which are invariant under residual gauge transformations (Eqs. (21, 49)) are, however, independent of these redundant orientational variables. For the following discussion, we focus on fields in the neighborhood of a monopole which we assume to be located at the origin. Once more, the starting point is the regularity of the Polyakov-loop variables ã before gauge fixing which allows for the Taylor expansion

$$
\tilde{\mathbf{a}}=M \mathbf{x}+\ldots
$$

In polar decomposition, the matrix $M$ is written as

$$
M=\rho m
$$

with a symmetric matrix $m$ and an orthogonal matrix $\rho$ with positive determinant. We have

$$
\frac{\partial^{2} \sin ^{2}\left(g \beta a_{0}\right)}{\partial x_{i} \partial x_{j}}=2\left(M^{T} M\right)_{i j}=2\left(m^{2}\right)_{i j} .
$$


Thus, the matrix $m$ is given in terms of the gauge invariant variables $a_{0}(\mathbf{x})$. Close to the origin, the Abelian magnetic field of the monopole (cf. Eq. (11)) is given by

$$
b_{k}=\frac{1}{2 g} \epsilon_{k i j} \sin \theta \partial_{i} \theta \partial_{j} \varphi \approx \frac{1}{2 g} \operatorname{det} m \frac{x_{k}}{\sin ^{3}\left(g \beta a_{0}\right)} .
$$

It is thus determined exclusively by the properties of $a_{0}(\mathbf{x})$.

More generally, the matrix $\rho$ of the decomposition (51) describes a rotation of the color coordinate system which can be accounted for by a residual gauge transformation (cf. Eqs. (48, 49)) and therefore does not affect gauge invariant quantities like the action (Eq. (47)). This can be seen in the following way. For fixed $m$, the orthogonal matrix $\rho$ can also be interpreted as a rotation of the spatial coordinate $\mathbf{y}=m \mathbf{x}$. If expressed in terms of $\mathbf{y}$, the fields $\mathbf{a}^{\mathrm{s}}$ and $\phi^{\mathrm{s}}$ are just rotated by $\rho$. As mentioned above this rotation can be undone by performing an appropriate residual gauge transformation (21).

Thus, in the neighborhood of the points $\mathrm{x}^{\mathrm{N}, \mathrm{S}}$ the singularities can be characterized completely by the gauge invariant Polyakov loop variables $a_{0}(\mathbf{x})$. This is trivially correct for those properties which are determined by general topological requirements. However our discussion also indicates that topological arguments are not sufficient to fully characterize the singularities. We illustrate this by rewriting the expression (53) for the magnetic field. Integration of Eq. (53) over a closed surface $\Sigma$ around $\mathbf{x}=\mathbf{0}$ yields the magnetic charge

$$
\int_{\Sigma} d^{2} \sigma_{i} b_{i}=\operatorname{sgn}(\operatorname{det} m) \frac{2 \pi}{g}
$$

which, as already follows from homotopy arguments, is independent of the details of the matrix $m$. Note that $b_{i}$ only carries one quantum of magnetic charge. Higher charges can occur when $M=0$ and higher terms in the Taylor expansion govern the behavior of a near the origin. We decompose the magnetic field as

$$
b_{k}=\frac{\operatorname{sgn}(\operatorname{det} m)}{2 g} \frac{x_{k}}{\left(\sum_{i} x_{i}^{2}\right)^{3 / 2}}+b_{k}^{\mathrm{tr}}
$$

where

$$
b_{k}^{\mathrm{tr}}=\frac{x_{k}}{2 g}\left[\frac{\operatorname{det} m}{\left(\sum_{i j}\left(m^{2}\right)_{i j} x_{i} x_{j}\right)^{3 / 2}}-\frac{\operatorname{sgn}(\operatorname{det} m)}{\left(\sum_{i} x_{i}^{2}\right)^{3 / 2}}\right] .
$$

By construction, the magnetic charge of $b_{k}^{\text {tr }}$ vanishes,

$$
\int_{\Sigma} d^{2} \sigma_{i} b_{i}^{\mathrm{tr}}=0
$$

Thus, $b_{k}^{\text {tr }}$ has identically vanishing divergence, i.e., it is a transverse singular field. This transverse field is in turn determined by currents which are generated by the charged components of the singular field configuration (11). For $\omega(x) \equiv 1$ or $\omega(x) \equiv \exp \left(2 i \pi x_{0} / \beta\right)$, the non-Abelian field strength has no spatial components which implies the transverse Abelian magnetic field to satisfy the Maxwell equation

$$
\operatorname{curl} \mathbf{b}^{\text {tr }}=g \mathbf{j} \quad \text { with } \quad \mathbf{j}=\operatorname{curl}\left(\boldsymbol{\Phi}^{\dagger} \times \boldsymbol{\Phi}\right) .
$$




\section{MONOPOLES AND INSTANTONS}

Up to this point our discussion has focused on the properties of the singularities which are generated in axial gauge if the Polyakov loop passes through the center of the group. In this section we establish a physically important sufficient condition for the presence of such singularities by relating the monopole charges associated with a certain field configuration to its topological charge. That such a relation must exist in the axial gauge is easily understood. As is well known, the topological charge

$$
\nu=\frac{g^{2}}{16 \pi^{2}} \int d^{4} x \operatorname{tr} F_{\mu \nu} \tilde{F}_{\mu \nu}, \quad \text { where } \quad \tilde{F}_{\mu \nu}=\frac{1}{2} \epsilon_{\mu \nu \rho \sigma} F_{\rho \sigma},
$$

can be written as a surface integral,

$$
\nu=\frac{g^{2}}{4 \pi^{2}} \int d^{3} \Sigma_{\mu} K_{\mu}
$$

with the topological current given by

$$
K_{\mu}=\epsilon_{\mu \nu \rho \sigma} \operatorname{tr}\left(\frac{1}{4} A_{\nu} F_{\rho \sigma}+\frac{i g}{6} A_{\nu} A_{\rho} A_{\sigma}\right) .
$$

In the axial gauge representation, the fields satisfy Abelian boundary conditions (see below) and a finite topological charge can thus arise only if singularities are present. In this case $\nu$ receives its full contribution from a surface surrounding the singularities.

More technically, to establish this relation we assume space to be a three-dimensional sphere. Generalizations to other compact manifolds or $\mathrm{R}^{3}$ are possible. We may cover $\mathrm{S}^{3}$ by two charts whose overlap does not contain any singularities (and where $P \neq \pm 1$ ). In order for the gauge fields on both charts to fulfill the gauge condition (5), the transition function relating the two charts must be Abelian and time independent and can therefore be continuously deformed to unity. The interpolating function can be used to transform the transition function away. Since the fields are furthermore periodic in time in the gauge chosen, we can, apart from the singularities, assume trivial boundary conditions. We furthermore assume that only generic singularities occur, i.e., points $\mathbf{x}_{i}$ where $P\left(\mathbf{x}_{i}\right)=z_{i}= \pm 1$ and strings $\mathbf{x}_{\alpha}(s)$ with $0 \leq s \leq 1$ which may either connect two singular points $\mathbf{x}_{i}$ or be closed. In the volume formula for the Pontrjagin index (59) we then exclude small regions around the singularities. The correct result will be recovered after shrinking these regions to zero volume, because the non-Abelian field strength is finite everywhere. In the surface integral (60), only the boundaries of the excluded regions will appear.

Because the singularities are time independent, $\mu$ in Eq. (60) is a spatial index. The second term in Eq. (61) therefore necessarily involves $a_{0}$,

$$
K_{j}^{A A A}=-\frac{i g}{2} \epsilon_{j k l} a_{0} \operatorname{tr} \tau_{3} A_{k} A_{l} .
$$

Under the trace, only the off-diagonal components of $A_{k}$ and $A_{l}$ contribute. These are finite at the strings and diverge linearly at the monopoles (cf. Eqs. (31, 10, 11)). At the monopoles only the quadratic singularity generated by the divergent parts of both field components contributes, 


$$
\begin{aligned}
\nu^{A A A} & =\frac{g^{2}}{4 \pi^{2}} \int d^{3} \sigma_{j} K_{j}^{A A A} \\
& =-\frac{i g}{8 \pi^{2}} \epsilon_{j k l} \int d^{3} \Sigma_{j} a_{0} \operatorname{tr} \tau_{3} \bar{\omega} \Omega_{\mathrm{D}} \partial_{k} \Omega_{\mathrm{D}}^{\dagger} \bar{\omega}^{\dagger} \bar{\omega} \Omega_{\mathrm{D}} \partial_{l} \Omega_{\mathrm{D}}^{\dagger} \bar{\omega}^{\dagger}
\end{aligned}
$$

The diagonal matrices $\bar{\omega}$ and $\bar{\omega}^{\dagger}$ cancel under the trace. The integration over surfaces oriented towards the monopoles splits into a (trivial) time integral and an integral over spheres around monopoles, which we choose to be oriented outwards:

$$
\begin{aligned}
\nu^{A A A} & =-\sum_{i} \frac{i g \beta a_{0}\left(\mathbf{x}_{i}\right)}{8 \pi^{2}} \epsilon_{j k l} \int_{\left|\mathbf{x}-\mathbf{x}_{i}\right|=\varepsilon} d^{2} \sigma_{j} \operatorname{tr} \tau_{3} \Omega_{\mathrm{D}} \partial_{k} \Omega_{\mathrm{D}}^{\dagger} \Omega_{\mathrm{D}} \partial_{l} \Omega_{\mathrm{D}}^{\dagger} \\
& =-\frac{1}{2} \sum_{\substack{i \\
P\left(\mathbf{x}_{i}\right)=-1}} n_{i},
\end{aligned}
$$

where we have identified the monopole winding number (24).

In the first term of Eq. (61), the field strength is finite because it transforms homogeneously under gauge transformations. Therefore, this term can at most diverge linearly and will contribute only at the strings. There, only the diagonal part of $A_{\nu}$ is singular (cf. Eq. (11)). In the field strength $F_{0 l}=\partial_{0} A_{l}-\partial_{l} a_{0} \tau_{3}-i g\left[a_{0} \tau_{3}, A_{l}\right]$, the commutator is purely off-diagonal and the first term integrates to zero because $A_{l}$ is periodic and the singular fields are time independent. Therefore, the contribution to the winding number reads

$$
\nu^{A F}=-\frac{g}{8 i \pi^{2}} \epsilon_{j k l} \int d^{3} \Sigma_{j} \operatorname{tr} \tau_{3} \bar{\omega} \Omega_{\mathrm{D}} \partial_{k} \Omega_{\mathrm{D}}^{\dagger} \bar{\omega}^{\dagger} \partial_{l} a_{0}
$$

Again, $\bar{\omega}$ and $\bar{\omega}^{\dagger}$ drop out. Inserting the explicit expression for the diagonal singular field, Eq. (11), we obtain

$$
\begin{aligned}
\nu^{A F} & =-\frac{g}{8 \pi^{2}} \epsilon_{j k l} \int d^{3} \Sigma_{j}(1+\cos \theta) \partial_{k} \varphi \partial_{l} a_{0} \\
& =\frac{g \beta}{2 \pi} \sum_{\alpha} n_{\alpha}\left(a_{0}\left(\mathbf{x}_{\alpha}(1)\right)-a_{0}\left(\mathbf{x}_{\alpha}(0)\right)\right)
\end{aligned}
$$

where the winding number carried by the strings, Eq. (26), has been inserted. Equation (66) shows that closed strings, for which $\mathbf{x}_{\alpha}(1)=\mathbf{x}_{\alpha}(0)$, do not contribute to the Pontrjagin index. Open strings must emanate from (end on) monopoles of the same (opposite) charge as the string. Therefore, Eq. (66) gives the same contribution as Eq. (64). If we further make use of the fact that on a compact manifold the total magnetic charge must vanish.' we can split this into contributions from northern and southern poles,

$$
\nu=\frac{1}{2}\left(\sum_{\substack{i \\ P\left(\mathbf{x}_{i}\right)=1}} n_{i}-\sum_{\substack{i \\ P\left(\mathbf{x}_{i}\right)=-1}} n_{i}\right)=\frac{1}{2} \sum_{i} z_{i} n_{i}
$$

\footnotetext{
${ }^{1}$ Non-trivial boundary conditions have been converted to singularities.
} 
i.e., the Pontrjagin index is determined by the two charges characterizing the monopoles (cf. Eqs. (13) and (24) $)$ and is given by the difference in the total charge of north $(P=1)$ and of south poles $(P=-1)$. Thus, an instanton configuration or a continuous deformation thereof must contain at least two magnetic monopoles. Indeed, it is straightforward to verify this relation for a periodic (finite-temperature) instanton. Explicit expressions are given in the next section. On the other hand, the presence of magnetic monopoles does not necessarily give rise to a topological charge. For instance, configurations describing oscillations of the Polyakov loop around the north pole which are most likely relevant for the deconfined phase generate a succession of $P=1$ poles and antipoles and thus do not contribute to the total charge at all. It is plausible that such oscillations around $P=1$ can be continuously deformed to a constant and the presence of two types of singularity is therefore required for a non-vanishing topological charge. The relation (67) actually displays the topological origin of the difference between field configurations containing only one or both types of singularity which has already been observed in our discussion of the expressions (46) for the field strength.

The above relation between instanton number and axial gauge monopoles has been derived before in a different framework [12] in which, however, not only the gauge field but also the non-Abelian field strength becomes singular. The coincidence of both results is remarkable since in our approach it is essential that the non-Abelian field strength transforms covariantly under the gauge fixing transformation (35).

\section{PHYSICS OF AXIAL GAUGE MONOPOLES - CONCLUSIONS}

We have presented a detailed analysis of the singular field configurations which occur when representing QCD in (modified) axial (temporal) gauge. (Most of our results actually also apply to the only partially fixed Polyakov gauge.) Singular gauge fields emerge as coordinate singularities when diagonalizing the (untraced) Polyakov loop or, equivalently, when defining the (local) color coordinate system by the direction of the Polyakov loop. This coordinate choice becomes ill-defined whenever the Polyakov loop is in the center of the group. At these points, the gauge fixed fields necessarily develop singularities. As is well known, homotopy arguments guarantee that, for a generic field configuration, the neutral Abelian field strength associated with the gauge fixed field configuration develops a monopole singularity. The complete and gauge covariant non-Abelian field strength must remain regular in the course of gauge fixing. Therefore, the charged components of the gauge fixed field configurations necessarily also develop singularities such that the singularities in the Abelian field strength are exactly canceled by the non-Abelian commutator. Furthermore, not only do the field configurations exhibit singular charged in addition to the singular Abelian components, these Abelian components in general also possess singularities in the transverse components in addition to the longitudinal ones. The presence of these singular transverse Abelian fields is not dictated by any topological requirement and, accordingly, their strength is arbitrary. Their sources are currents generated by the singular charged fields.

Although we have performed this detailed study of the structure of the singular fields for a particular gauge choice, the arguments directly apply whenever gauge fixing is achieved by 
diagonalization of a local quantity in the adjoint representation. Locally the structure of the singular fields as derived above remains valid for other gauges of this type. Globally, however, the fields are in general not independent of one of the space-time coordinates. Despite this common structure of the singularities, the physics consequences must be expected to be quite different for different gauge choices. This is indicated by the connection which we have derived between monopole charges and the instanton number. The presence of trivial boundary conditions in the gauge fixed formulation is essential in establishing a relation between instantons and monopoles. The necessity of the presence of two distinct types of singularity (north and south pole) points to properties of this relation which are specific for axial type gauges in which a group element is actually diagonalized. Finally, the simultaneous role of the Polyakov loop in characterizing confined and deconfined phases on the one hand and in determining positions and strengths of the singular gauge fields on the other, is obviously a very particular property of the axial gauge which we shall exploit in this concluding paragraph.

The central result of our investigations is summarized in the expression for the axial gauge QCD partition function

$$
Z=\sum_{\mathbf{n}} Z_{\mathbf{n}}=\sum_{\mathbf{n}} \int D\left[a_{0}^{\mathbf{n}}\right] \int \prod_{i=1}^{3} D\left[\hat{A}_{i}\right] e^{-S[\hat{A}+\alpha]} .
$$

The sum over field configurations has been decomposed according to the number $\mathbf{n}=$ $\left(n_{\mathrm{N}}, n_{\mathrm{S}}\right)$ of north $\left(n_{\mathrm{N}}\right)$ and south $\left(n_{\mathrm{S}}\right)$ pole singularities; i.e., the path integral in $Z_{\mathbf{n}}$ is performed over field configurations in which the Polyakov loop passes $n_{\mathrm{N}, \mathrm{S}}$ times through the north and south pole, respectively. For this decomposition to be meaningful, regularization of the generating functional is required. According to our analysis above, the singular component $\alpha$ (cf. Eq. (42)) is completely determined by the Polyakov loop variables,

$$
\alpha=\alpha\left[a_{0}^{\mathbf{n}}\right] .
$$

The quantum fluctuations $\hat{A}$ around the singular field configurations give rise to infinite values of the action unless they satisfy the boundary conditions (37). In the generating functional $A_{0}$ has been eliminated up to the gauge invariant Polyakov loop variables. Apart from singularities, this elimination also generates a non-trivial but explicitly calculable measure $D\left[a_{0}^{\mathbf{n}}\right]$, the Haar measure of $\mathrm{SU}(2)$ (cf. [13]). The action is easily calculated in terms of singular and regular fields by using the explicit expressions (44, 46) for the field strength components. (The inclusion of quarks into the formalism is straightforward since it does not affect the gauge fixing procedure.)

Starting from the above generating functional, a dynamical picture can be developed by integrating out the Polyakov loop variables. Application of techniques developed for the $\mathbf{n}=(0,0)$ sector in Ref. [17] will yield an effective theory of QCD with $A_{0}$ completely removed and with the other degrees of freedom being coupled to the singular field configurations discussed above. In a qualitative discussion we now attempt to anticipate some of the main properties of such a formulation of QCD. We first discuss issues concerning the number of singularities typically involved in a generic field configuration starting with a brief discussion of the Polyakov loop associated with instantons. This will lead to an estimate for the 
monopole density associated with a dilute gas of instantons. Explicit expressions for finite temperature instantons of size $\lambda$ are given in Ref. [20],

$$
A_{\mu}=\frac{1}{g} \bar{\sigma}_{\mu \nu} \partial_{\nu} \ln \left\{1+\frac{(\gamma / u) \sinh u}{\cosh u-\cos v}\right\},
$$

where

$$
u=2 \pi\left|\mathbf{x}-\mathbf{x}_{0}\right| / \beta, \quad v=2 \pi t / \beta, \quad \gamma=2(\pi \lambda / \beta)^{2} .
$$

Using these expressions, the associated Polyakov loops are easily calculated,

$$
P(\mathbf{x})=\exp \left\{i \pi \frac{\left(\mathbf{x}-\mathbf{x}_{0}\right) \cdot \boldsymbol{\tau}}{\left|\mathbf{x}-\mathbf{x}_{0}\right|} \chi(u)\right\}
$$

The function

$$
\chi(u)=1-\frac{\left(1-\gamma / u^{2}\right) \sinh u+(\gamma / u) \cosh u}{\sqrt{(\cosh u+(\gamma / u) \sinh u)^{2}-1}}
$$

decreases monotonically from the value 1 at $u=0$ with increasing $u$ and vanishes at $u=\infty$. Thus, the Polyakov loop associated with an instanton passes through the south pole at $u=0$ (the center of the instanton) and approaches the north pole at asymptotic distances. As is well known, instantons carry one unit of topological charge which determines the value $8 \pi^{2} / g^{2}$ of the action. We have seen above that with both north and south poles occurring in a certain field configuration the corresponding magnetic field strength is necessarily different from zero $(|\omega(x)| \neq 1$ in Eq. (46)). The necessarily finite value of the action is minimized if the choice of $\omega(x)$ corresponds to an instanton.

Results of the instanton liquid model [21] and of lattice QCD [22] suggest the presence of a finite density $n_{\mathrm{I}}$ of instantons in the confined phase. In order to relate instanton and monopole densities, we treat the instantons as independent; in this case the Polyakov loop is given by a product over the Polyakov loops associated with single instantons (or antiinstantons). For small instanton sizes $\lambda \ll \beta$, the number of instantons plus anti-instantons integrated over in the time integral of the Polyakov loop for fixed $\mathbf{x}$ is of the order of $\beta n_{\mathrm{I}} \lambda^{3}$. On the average, the number of instantons and anti-instantons will be the same and thus the phase accumulated in the time integral will be given by the fluctuations and is thus expected to be of the order of $\pi \sqrt{\beta n_{\mathrm{I}} \lambda^{3}}$. When changing the position $\mathbf{x}$ by an instanton size of $\lambda$ we expect, in the absence of correlations between the instantons, a different value of the phase which however is of the same order of magnitude. Thus, in this change of $\mathbf{x}$ the Polyakov loop will typically pass $\sqrt{\beta n_{\mathrm{I}} \lambda^{3}}$ times through the center of the group which implies the following estimate for the monopole density (counting both poles and antipoles) at low temperatures

$$
n_{\mathrm{M}} \propto\left(\beta n_{\mathrm{I}} \lambda\right)^{3 / 2}, \quad \lambda \ll \beta,
$$

while for high temperatures, the integral involves at most one instanton and we therefore expect 


$$
n_{\mathrm{M}} \propto \beta n_{\mathrm{I}}, \quad \lambda \geq \beta .
$$

Surprisingly, the result (74) implies an infinite monopole density in the zero temperature limit. Although derived on the basis of a finite value of the instanton density, this result is to a large extent independent of the particular model. Let us consider more generally a field configuration with significant variations on length scales $\geq \lambda_{1}$ before gauge fixing. Using similar arguments as above, such a configuration will typically lead to a value of $\pi \sqrt{\beta / \lambda_{1}}$ for the phase of the Polyakov loop and if the field configurations are correlated over distances $\lambda_{2}$ the value $\left(\beta / \lambda_{1} \lambda_{2}^{2}\right)^{3 / 2}$ for the monopole density follows. The characteristic $\beta^{3 / 2}$ dependence is mostly a consequence of the integration over the time interval which becomes ill-defined in the zero temperature limit. It thus appears that irrespective of the temperature, axial gauge monopoles condense. Even a gas of smooth field configurations with small values of the action gives rise to a finite and in the zero temperature limit divergent monopole density.

Beyond condensation of magnetic monopoles via instantons, the system has the additional option of condensation involving one type (north or south) of poles and corresponding antipoles only. As our discussion of the singularity structure shows, such field configurations do not require non-vanishing magnetic field strength and the electric field contribution to the action can be made arbitrarily small for sufficiently smooth Polyakov loop variables. Thus, suppression of such field configurations could only come through their coupling to the quantum fluctuations. Since it appears that instantons may not be able to account for confinement 23] or, more precisely, for a realistic value of the string constant [22], this additional option could be relevant and, by a correspondingly increased monopole density, generate the missing strength for the dual Meissner effect.

In axial gauge, condensation of monopoles must be expected to persist beyond the deconfinement transition. With the center symmetry broken, the Polyakov loop is not distributed symmetrically around the equator of $\mathrm{S}^{3}$ any more. It rather approaches more and more either the north or the south pole with increasing temperature. An expectation value $P \neq \pm 1$ in the infinite temperature limit is compatible neither with the Stefan-Boltzmann law (cf. [24]) nor with the expected dimensional reduction to 2+1-dimensional QCD (cf. [17]). Thus, as the Polyakov loop approaches one of the poles, the probability to pass through this pole and therefore the monopole density must be expected to increase. On the other hand, for this increased density to be compatible with perturbation theory and, in particular, not to lead to confinement, one might expect poles and antipoles to compensate each other to a large extent. This would be the case if poles and antipoles are strongly correlated with each other. We thus expect the high-temperature phase to consist of a gas of magnetic dipoles.

So far our qualitative arguments have dealt mainly with the entropy associated with a finite monopole density. Dynamics has been involved only implicitly when invoking the instanton gas picture. In concluding we now present qualitative arguments concerning basic dynamical issues. As our formal development shows, singular monopole configurations can have vanishing magnetic field energy and may contribute only little to the action (cf. Eq. (46)). For this reason we focus on the coupling of the quantum fluctuations to the singular fields which is contained in the covariant derivative in Eq. (45). In the presence of singularities quantum fluctuations have to satisfy the conditions (37), otherwise infinite action results; this condition just expresses finiteness of the action before gauge fixing. Given a finite monopole density, and assuming no particular correlations between the monopoles, this 
condition cannot be satisfied for long-wavelength fluctuations, i.e., we expect fluctuations with wave number

$$
k \leq k_{\min }=n_{\mathrm{M}}^{1 / 3}
$$

to be dynamically suppressed. On the other hand, long-wavelength excitations associated with bilinears such as $\boldsymbol{\Phi}^{\dagger}(x) \cdot \boldsymbol{\Phi}(x)$ which might be interpreted as two-gluon states are not necessarily affected by the continuity conditions and may be associated with excitation energies which remain finite at $T=0$. Thus, a high density of monopoles indeed seems to generate confinementlike phenomena. At high temperatures, appropriate correlations between poles and antipoles must make this mechanism ineffective.

In order to further illustrate effects of the coupling to magnetic monopoles, we consider the following contribution to the action generated by the coupling of the charged quantum fluctuations to the Abelian monopole fields via the four-gluon vertex,

$$
\delta S=-g^{2} \int d^{4} x \sum_{i=1}^{3} \hat{\boldsymbol{\Phi}}^{\dagger}(x) \cdot \hat{\mathbf{\Phi}}(x) \mathbf{a}^{\mathrm{s}}(\mathbf{x}) \cdot \mathbf{a}^{\mathrm{s}}(\mathbf{x}) .
$$

At long wavelengths the quantum fluctuations thus acquire a mass $\delta m$ by coupling to the Abelian magnetic field,

$$
\delta m^{2}=g^{2} \frac{1}{V} \int_{V} d^{3} x \mathbf{a}^{\mathrm{s}}(\mathbf{x}) \cdot \mathbf{a}^{\mathrm{s}}(\mathbf{x})
$$

To compute this mass term we assume the singular field $\mathbf{a}^{\mathrm{s}}(\mathbf{x})$ to be given by a superposition of the standard monopole fields of Eq. (15),

$$
\mathbf{a}^{\mathrm{S}}(\mathbf{x})=\sum_{i=1}^{N} \mathbf{a}^{n_{i}}\left(\mathbf{x}-\mathbf{x}_{i}\right)
$$

with positions $\mathbf{x}_{i}$ of the monopoles and charges $n_{i}= \pm 1$ and vanishing total magnetic charge,

$$
\sum_{i=1}^{N} n_{i}=0
$$

In this way the functional integral over the Polyakov loop variables in Eq. (68) has effectively been replaced by a summation over positions $\mathbf{x}_{i}$ and charges $n_{i}$ of these prescribed representative fields.

Using standard identities from electrostatics, the mass term can be written as

$$
\delta m^{2}=\frac{1}{4 V} \int_{V} d^{3} x \sum_{i, j=1}^{N} \frac{n_{i} n_{j}}{\left|\mathbf{x}-\mathbf{x}_{i}\right|\left|\mathbf{x}-\mathbf{x}_{j}\right|}=-\frac{\pi}{V} \sum_{\substack{i, j=1 \\ i<j}}^{N} n_{i} n_{j}\left|\mathbf{x}_{i}-\mathbf{x}_{j}\right| .
$$

Similarly, neutral gauge field fluctuations acquire a mass $\delta m_{3}$ by coupling to the singular charged components,

$$
\delta m_{3}^{2}=g^{2} \frac{1}{V} \int_{V} d^{3} x \boldsymbol{\phi}^{\mathrm{s}}(\mathbf{x}) \cdot \boldsymbol{\phi}^{\mathrm{s}}(\mathbf{x})
$$


Evaluation of this expression by using the charged partner (Eq. (17)) of the Dirac monopole field yields

$$
\delta m_{3}^{2}=\delta m^{2}
$$

Equation (81) displays the resistance of the system to proliferate production of monopoles. Furthermore since $\delta m^{2}$ decreases with decreasing "distance" between monopoles, coupling to quantum fluctuations effectively induces an attractive interaction between monopoles and antimonopoles which, if strong enough, leads to monopole-antimonopole annihilation and ultimately to a vanishing $\delta m^{2}$. Also note the confining nature of the interaction between magnetic monopoles of opposite charge which is due to the standard Meissner effect with the field $\hat{\boldsymbol{\Phi}}$ playing the role of the Higgs field. If still valid in the confining phase, this perturbative treatment would point to a gluon mass which, with decreasing temperature, increases with the monopole density (cf. Eq. (74) ). Without specifying the distribution of the monopoles, a reliable estimate of the gluon mass is not possible. At high temperatures, as argued above, we might expect the dynamics to favor monopoles of opposite charge to be bound in dipoles. If the extension of the dipoles is much smaller than their separation, the expression (81) can be simplified and yields a gluon mass

$$
\delta m^{2}=n_{\mathrm{M}} d
$$

determined by the monopole density and the average dipole size $d$. In such a description of the high temperature phase as a gas of strongly correlated magnetic monopoles, the appearance of a magnetic gluon mass is rather natural. Temperature or coupling constant dependence of such a magnetic mass are implicitly determined by monopole density and dipole size and cannot be obtained by these simple arguments.

The picture of the monopole dynamics which we have developed is of some relevance for the interpretation of results obtained in QCD lattice calculations. The axial gauge discussed here is closely related to the Polyakov gauge employed in lattice calculations. If the full local Abelian gauge symmetry still present in the Polyakov gauge is fixed by eliminating $A_{0}^{3}(x)$ up to the zero modes $a_{0}^{3}(\mathbf{x})$, the axial gauge representation is obtained. The properties of the monopoles are identical in these two gauges. When comparing our qualitative arguments with numerical results [5] we find support for persistence of a monopole condensate beyond the deconfinement transition. It is not known, though suggested by calculations for 2+1-dimensional QCD [25], whether the strong correlations between poles and antipoles as indicated by our arguments indeed exist in the high temperature phase nor has a relation between magnetic mass and monopole properties like Eq. (84) been established numerically. Such results would be helpful for clarifying non-perturbative properties of the high temperature phase. Lattice calculations exhibit strong dependence in the monopole dynamics on the particular gauge choice. In the maximal Abelian gauge, a significant monopole density is present only in the confined phase [26,27,6]. This qualitative difference between the axial or Polyakov gauge, on the one hand, and the maximal Abelian gauge on the other may be due to the very different properties of the gauge condition in the weak coupling limit. The differential form of the maximal Abelian gauge condition [4.5],

$$
\left(\partial_{\mu}+i g A_{\mu}^{3}\right) \Phi_{\mu}=0,
$$


reduces for weak coupling to the standard Lorentz gauge. This gauge is (up to overall zero modes) well defined. In the absence of gauge ambiguities no monopoles are present. On the other hand, in the same weak coupling limit, the Polyakov loop approaches the north pole

$$
P(\mathbf{x}) \rightarrow \mathbb{1} \quad \text { for } \quad g \rightarrow 0,
$$

i.e., space gets filled with static monopoles in axial or Polyakov gauge. This suggests the possibility of a unified picture of monopole dynamics for the confined phase only and monopole condensation as a signature of confinement only in gauges which become well defined in the weak coupling limit.

In summary, we have identified in this qualitative discussion of the physics of axial gauge monopoles two conflicting tendencies of monopole dynamics. On the one side, entropy arguments naturally favor production of monopoles. Our discussion moreover suggests an unusual increase in entropy with decreasing temperature. On the other side, monopole interactions mediated by coupling to quantum fluctuations induce attractive monopole-antimonopole interactions which ultimately will induce annihilation of monopole-antimonopole pairs. These two conflicting tendencies suggest an interpretation of the confined phase as a phase with a finite and at $T=0$ divergent density of weakly correlated monopoles, while in the deconfined phase, monopoles are bound into dipoles. The confinement-deconfinement transition is thus similar to the phase transition in the two-dimensional XY model which occurs by vortex unbinding, however with high and low temperature phases interchanged. Unlike in standard systems, in QCD the entropy associated with monopoles increases with decreasing temperature which might be ultimately the source of the unusual phenomenon of spontaneous symmetry breakdown in the high temperature phase.

\section{ACKNOWLEDGMENTS}

This work has been supported by the Bundesministerium für Bildung, Wissenschaft, Forschung und Technologie and the Monbusho International Scientific Research Program (No. 06044053). We would like to thank M. Thies for his collaboration in the early stage of this work, U.-J. Wiese and K. Yazaki for fruitful discussions. We express our gratitude to V. Eletsky, A. Kalloniatis and L. v. Smekal for careful reading of the manuscript and valuable comments and suggestions concerning both physics and presentation. F.L. thanks K. Yazaki for the hospitality extended to him at the University of Tokyo where part of this work was done. 


\section{REFERENCES}

[1] I. M. Singer, Commun. Math. Phys. 60, 7 (1978).

[2] V. N. Gribov, Nucl. Phys. B139, 1 (1978).

[3] S. Mandelstam, Phys. Rep. 67, 109 (1980).

[4] G. 't Hooft, Nucl. Phys. B190, 455 (1981).

[5] A. S. Kronfeld, G. Schierholz, and U.-J. Wiese, Nucl. Phys. B293, 461 (1987).

[6] M. I. Polikarpov, Nucl. Phys. B (Proc. Suppl.) 53, 134 (1997).

[7] Z. F. Ezawa and A. Iwazaki, Phys. Rev. D 25, 2681 (1982).

[8] T. Suzuki and I. Yotsuyanagi, Phys. Rev. D 42, 4257 (1990).

[9] M. N. Chernodub and F. V. Gubarev, JETP Lett. 62, 100 (1995).

[10] R. C. Brower, K. N. Orginos, and C.-I. Tan, Phys. Rev. D 55, 6313 (1997).

[11] T. T. Wu and C. N. Yang, Nucl. Phys. B107, 365 (1976).

[12] H. Reinhardt, Nucl. Phys. B503, 505 (1997).

[13] F. Lenz, H. W. L. Naus, and M. Thies, Ann. Phys. (N.Y.) 233, 317 (1994).

[14] P. A. M. Dirac, Proc. R. Soc. London A133, 60 (1931).

[15] H. Grießhammer, Ph.D. thesis, University of Erlangen, 1996; hep-ph/9709462.

[16] K. Langfeld, H. Reinhardt, and M. Quandt, hep-th/9610213.

[17] F. Lenz and M. Thies, hep-th/9802066.

[18] A. Frenkel and P. Hrasko, Ann. Phys. (N.Y.) 105, 288 (1977).

[19] M. Blau and G. Thompson, Commun. Math. Phys. 171, 639 (1995).

[20] B. J. Harrington and H. K. Shepard, Phys. Rev. D 17, 2122 (1978).

[21] T. Schäfer and E. V. Shuryak, Rev. Mod. Phys. 70, 323 (1998).

[22] M. C. Chu, J. M. Grandy, S. Huang, and J. W. Negele, Phys. Rev. D 49, 6039 (1994).

[23] C. G. Callan, R. F. Dashen, and D. J. Gross, Phys. Rev. D 17, 2717 (1978).

[24] V. L. Eletsky, A. C. Kalloniatis, F. Lenz, and M. Thies, Phys. Rev. D 57, 5010 (1998).

[25] H. D. Trottier, G. I. Poulis, and R. M. Woloshyn, Phys. Rev. D 51, 2398 (1995).

[26] A. S. Kronfeld, M. L. Laursen, G. Schierholz, and U.-J. Wiese, Phys. Lett. 198B, 516 (1987).

[27] T. Suzuki, Nucl. Phys. B (Proc. Suppl.) 30, 176 (1993). 